\title{
Parameter Estimation of the Burr Type XII Distribution with a Progressively Interval-Censored Scheme Using Genetic Algorithm
}

\author{
Tzong-Ru Tsai ${ }^{\mathrm{a}^{*}}$, Jyun-You Jiang ${ }^{\mathrm{b}}$, Yuhlong Lio ${ }^{\mathrm{c}}$, Nan Jiang ${ }^{\mathrm{c}}$ and Ya-Yen Fan ${ }^{\mathrm{a}}$

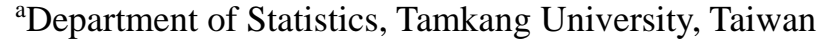 \\ ${ }^{b}$ School of Statistics, Southwestern University of Finance and Economics, China \\ ${ }^{\mathrm{c}}$ Department of Mathematical Sciences, University of South Dakota, USA \\ *Corresponding Author: trtsai@stat.tku.edu.tw
}

\begin{abstract}
Burr type XII distribution (BXIID) has been widely used to model lifetime data sets. The flexibility of the BXIID is established due to its two shape parameters. To save test time and cost, the BXIID parameters can be inferred by using the maximum likelihood estimation method based on a date set with incomplete lifetime information. But the maximum likelihood estimates (MLEs) of BXIID parameters could have a big bias and mean squared error (MSE) if the sample size is small or the MLEs are evaluated with improper initial parameters. In this study, a progressively interval-censored (PIC) scheme is used to implement the life test, and the Genetic Algorithm (GA) is applied to reduce the bias and MSEs of the MLEs of the BXIID parameters. An extensive Monte Carlo simulation was conducted to evaluate the estimation performance of the typical maximum likelihood estimation method (TMLEM) and GA. Simulation results show that the GA is competitive with the TMLEM in terms of resulting in a smaller bias and MSE in parameter estimation.
\end{abstract}

Keywords: Burr type XII distribution; Genetic Algorithm; maximum likelihood estimation; Monte Carlo simulation; progressively interval-censored scheme.

\section{Abbreviations and Acronyms}

BXIID Burr type XII distribution

MSE mean squared error

MLE maximum likelihood estimate

GA genetic algorithm
PIC progressively interval-censored

TMLEM typical maximum likelihood estimation method

\section{Notations}

$y \quad$ BXIID lifetime

$\boldsymbol{f}(\boldsymbol{y})$ density function of $\boldsymbol{y}$

$\boldsymbol{F}(\boldsymbol{y}) \quad$ distribution function of $\boldsymbol{y}$

$\boldsymbol{t}_{\boldsymbol{i}} \quad i^{\text {th }}$ inspection time

$x_{i} \quad$ number of failed subjects in $\left(t_{i-1}, t_{i}\right]$

$\boldsymbol{R}_{\boldsymbol{i}} \quad$ number of surviving subjects removed at $\boldsymbol{t}_{\boldsymbol{i}}$

$\boldsymbol{L}($.$) \quad likelihood function$

$\boldsymbol{\ell}($.$) \quad log-likelihood function$

$\hat{\boldsymbol{c}}_{\boldsymbol{M}} \quad$ MLE of $\boldsymbol{c}$ obtained using TMLEM

$\widehat{\boldsymbol{k}}_{\boldsymbol{M}} \quad$ MLE of $\boldsymbol{k}$ obtained using TMLEM

$\hat{\boldsymbol{c}}_{\boldsymbol{G}} \quad$ MLE of $\boldsymbol{c}$ obtained using GA

$\widehat{\boldsymbol{k}}_{\boldsymbol{G}} \quad$ MLE of $\boldsymbol{k}$ obtained using GA

$\boldsymbol{x} . \boldsymbol{s} \quad$ generated value of $\boldsymbol{x}$ in simulation

$\boldsymbol{r} . \boldsymbol{s} \quad$ generated value of $\boldsymbol{R}$ in simulation

$\delta_{i} \quad\left[F\left(t_{i}\right)-F\left(t_{i-1}\right)\right] /\left[1-F\left(t_{i-1}\right)\right]$

$\lfloor z\rfloor \quad$ largest integer equal or smaller than $z$ 
Tadikamalla [2] showed that the BXIID connects to lots of useful lifetime distributions. He concluded that the BXIID can be used to model almost all data sets. Many existing studies considered the BXIID as a favorable baseline distribution for performing reliability assessment [3]-[16]. The density function and distribution function of BXIID are, respectively, defined by

$$
f(x \mid c, k)=c k x^{c-1}\left(1+x^{c}\right)^{-k-1},
$$

and

$$
F(x \mid c, k)=1-\left(1+x^{c}\right)^{-k}, x>0, c>0, k>0
$$

where $c$ and $k$ are both shape parameters.

Because the duration to collect a complete data set of failure times of highly reliable products is time consuming and could not be affordable by the manufacturers. Some schemes for saving testing time, such as censoring and truncated schemes, have been proposed to conduct the life test, and then a censoring sample or truncated sample can be used to implement reliability assessment for highly reliable products.

Censoring often is implemented with a time censoring scheme, failure censoring scheme, or interval censoring scheme. The time censoring is type I censoring, which terminates the life test at a predetermined time. The observed failure times and the termination time are used for the type I censored sample to implement reliability assessment. The failure censoring is type II censoring, a life test under the type II censoring is terminated when a predetermined number of failure times is collected. All the failure times and the last surviving time are used for the type II censored sample to implement reliability assessment.

The number of failed subjected in a type I censoring scheme is a random variable, and the termination time in a type II censoring scheme is a random variable. The type I censoring scheme is favorable by practitioner because the termination time of life test can be predetermined. However, the practitioner may only inspect the life test at specific times, and only the number of failed subjects in individual time intervals are taken into account. The exact failure times of subjects are unknown. Such inspection method is named interval-censoring method. The termination time of life test also can be predetermined by using an interval-censoring scheme, and such scheme can be conducted easier than conducting a type I censoring.

To avoid only observing the shortest lifetimes of subjects in a life test, the practitioner would like to occasionally removing some surviving subjects during the life test. In this paper, an interval-censoring scheme of constant removals, named a PIC scheme, is taken for the life test to implement reliability assessment for highly reliable products. Progressively censoring schemes have been widely used for life testing [8], [11]-[13], [15] and [17].

A PIC sample is collected as follows: $n$ subjects are subjected to life testing at the initial time $t_{0}=0$. The number of failed subjects are taken into account at the scheduled times $t_{1}, t_{2}, \ldots, t_{m}$, respectively, and $R_{i}$ surviving subjects are randomly removed from the life test at time $t_{i}$. The life test terminates at time $t_{m}$. Let $x_{i}$ denote the number of subjects failed in the interval $\left(t_{i-1}, t_{i}\right]$. Aggarwala [17] derived the likelihood function, based on a PIC sample, by

$$
\begin{gathered}
L(\mathrm{c}, \mathrm{k}) \propto \prod_{i=1}^{m}\left[F\left(t_{i} \mid c, k\right)-F\left(t_{i-1} \mid c, k\right)\right]^{x_{i}}[1- \\
\left.F\left(t_{i} \mid c, k\right)\right]^{R_{i}},
\end{gathered}
$$

where $F\left(t_{0} \mid c, k\right)=0$. No closed forms of solutions for Equation (3) can be found. Iterative numerical search methods are suggested to search for the MLEs of parameters. The MLEs of $c$ and $k$, denoted by $\hat{c}_{M}$ and $\hat{k}_{M}$, can be obtained, by using the TMLEM, as follows:

$$
\left(\hat{c}_{M}, \hat{k}_{M}\right)=\underset{c, k>0}{\operatorname{aug} \max } \ell(c, k),
$$

where $\ell(c, k)=\log L(c, k)$ denotes the log-likelihood function. Two difficulties for using (4) to obtain the MLEs:

1. As the true values of $c$ or $k$ close to 0, the TMLEM often fails due to the values of $\log (c)$ and $\log (k)$ could be undefined to maximize the log-likelihood function.

2. Because initial parameters of $c$ and $k$ need to be predetermined for the TMLEM, the TMLEM is sensitive to the initial parameters. The identification of initial parameters is difficult to do if full knowledge about the lifetime data is not complete, or too many multiple parameters need to be solved simultaneously.

To overcome these two difficulties of using the TMLEM to find the MLEs of the parameters of the BXIID based on a PIC sample, GA can act a potential candidate method to search the MLEs of the parameters $c$ and $k$ of the BXIID. This study denotes the MLEs of $c$ and $k$, obtained by using the GA, by $\hat{c}_{G}$ and $\hat{k}_{G}$, respectively. The principal of $\mathrm{GA}$ is briefly given as follows: 
1. Choose an initial population.

2. Determine the fitness of each individual.

3. Perform selection.

4. Perform crossover and mutation

5. Determine the fitness of each individual.

6. Perform selection.

7. The generational process is repeated until a termination condition has been reached.

Common termination condition(s) can be one of the following condition, or combinations of them:

1. A solution is reached to meet the specific criteria.

2. The fixed number of generations is reached.

3. The allocated budget is reached.

4. The highest ranking solution's fitness is reached, or the solutions cannot be improved by successive iterations.

The GA is used to find the MLEs of $c$ and $k$ to maximize (3) not to maximize the log-likelihood function. Moreover, the GA does not need to set up initial parameters for obtaining the MLEs. Numerous existing studies about using GA for computation can be found [18]-[25].

The rest of this paper is organized as follows. In Section 2, an extensive Monte Carlo simulation was conducted to compare the estimation performance of the TMLEM and GA for PIC samples, which were generated from the baseline of BXIID based on an algorithm. Conclusions drawn from this study are provided in Section 3

\section{Monte Carlo Simulation}

The simulation, using the TMLEM and GA to obtain the MLEs of the parameters of the BXIID, was conducted with two algorithms. Algorithm 1 is provided to generate PIC samples, each sample contains $m$ failed subjects and removals, denoted by $\left(x_{1}, x_{2}, \ldots, x_{m}\right)$ and $\left(R_{1}, R_{2}, \ldots, R_{m}\right)$. The MLEs of the BXIID distribution parameters are evaluated by using the generated PIC samples. The construction of Algorithm 1 is similar to the algorithm provided by Aggarwala [17].

\section{Algorithm 1.}

Step 1. Let $x_{0}=R_{0}=0$, Determine the inspection times

\footnotetext{
1 An instruction to employ the R package "optim” can be found via the link http://127.0.0.1:29635/library/stats/html/optim.html.
}

by $t_{1}, t_{2}, \ldots, t_{m}$ and withdraw probabilities by $p_{1}, p_{2}, \ldots, p_{m-1}, p_{m}$, where $0 \leq p_{j}<1$ for $j=$ $1,2, \ldots,(m-1)$, and $p_{m}=1$;

Step 2. Let $i=0$ and x.s $=r . s=0$;

Step 3. Let $i=i+1$; Generate $x_{i}$ from the binomial distribution with sample size $(n-x . s-r . s)$ and the success probability

$$
\delta_{i}=\frac{F\left(t_{i} \mid c, k\right)-F\left(t_{i-1} \mid c, k\right)}{1-F\left(t_{i-1} \mid c, k\right)}
$$

Let $R_{i}=\left\lfloor p_{i} \times\left(n-\sum_{j=1}^{i-1}\left(x_{j}+R_{j}\right)-x_{i}\right)\right\rfloor$, where $\lfloor z\rfloor$ is the largest integer equal or smaller than $z$; Step 4. Let $x . s=\left(x . s+x_{i}\right)$ and r.s $=\left(r . s+R_{i}\right)$; Step 5. If $i<m$, revert to Step 3; otherwise stop the algorithm.

The R package “optim" ${ }^{1}$ provides a general-purpose optimization based on Nelder-Mead, quasi-Newton and conjugate-gradient algorithms, and the optimization procedure includes an option for box-constrained optimization and simulated annealing. In this paper, the $\mathrm{R}$ package "optim" was used to evaluate the MLEs of the parameters of the BXIID for the TMLEM.

Because the target function in (3) is nonlinear and complicated, search the MLEs of parameters by using the TMLEM could be failed. In this study the MLEs are also searched by using the GA over the parameter space. The $\mathrm{R}$ package "GA"2, which was published in 2014, was used to implement the GA procedure. The package “GA” provides a flexible general-purpose set of tools for implementing GA search in both the continuous and discrete case, whether constrained or not. The simulation was conducted using the algorithm 2 .

\section{Algorithm 2.}

Step 1. Generating a PIC sample of $\left(x_{1}, x_{2}, \ldots, x_{m}\right)$ and $\left(R_{1}, R_{2}, \ldots, R_{m}\right)$ for the baseline BXIID, which defined by (1) and (2), using Algorithm 1.

Step 2. Obtain the MLEs $\left(\hat{c}_{M}, \hat{k}_{M}\right)$ and $\left(\hat{c}_{G}, \hat{k}_{G}\right)$ by using the TMLEM with given initial parameters of $(c, k)$ and GA.

Step 3. Repeat Steps 1-2 1000 times, and denote the resulting MLEs by $\left(\hat{c}_{M, i}, \hat{k}_{M, i}\right)$ and $\left(\hat{c}_{G, i}, \hat{k}_{G, i}\right)$ for $i=$ $1,2, \ldots, 1000$. Evaluating the bias and MSEs of the

\footnotetext{
2 See the menu of using R package GA for more information via the link http://cran.rproject.org/web/packages/GA/.
} 
estimators, respectively, by

$$
\text { Bias }=\left(\frac{1}{1000} \sum_{i=1}^{1000} \hat{\theta}_{i}\right)-\theta,
$$

and

$$
\operatorname{MSE}=\frac{1}{1000} \sum_{i=1}^{1000}\left(\hat{\theta}_{i}-\theta\right)^{2}
$$

where $\hat{\theta}_{i}$ denotes the MLE of the parameter $\theta$ for the the simulation run $i$. If $\theta=c$, then $\hat{\theta}_{i}$ can be $\hat{c}_{M, i}$ or $\hat{c}_{G, i}$; and if $\theta=k$, then $\hat{\theta}_{i}$ can be $\hat{k}_{M, i}$ or $\hat{k}_{G, i}$.

The parameters, population size 50, probability of crossover 0.8 , probability of mutation 0.1 , and the iterations 100 , are used to implement the GA.

Table 1. Bias and MSEs of the MLEs of the parameters $c$ and $k$

\begin{tabular}{|c|r|r|r|r|}
\hline & \multicolumn{1}{|c|}{$\operatorname{Bias}(c)$} & \multicolumn{1}{c|}{$\operatorname{MSE}(c)$} & $\operatorname{Bias}(k)$ & $\operatorname{MSE}(k)$ \\
\hline GA & 2.645 & 15.847 & 2.304 & 12.987 \\
\hline TMLEM & 4.876 & 133.417 & 3.370 & 41.934 \\
\hline
\end{tabular}

True parameters $(c, k)=(3,5), n=30, m=5, p_{i}=$ 0.05 for $i=1,2,3,4$ and $p_{5}=1$ were given for the BXIID in this simulation study. The common initial parameters $\left(c_{0}, k_{0}\right)=(1,1)$ are used for the TMLEM method, and the GA search the MLEs of parameters over the interval of $(0.01,10)$. All simulation results were prepared in Table 1, Fig. 1 and 2. Table 1 shows that the MLEs $\hat{c}_{G}$ and $\hat{k}_{G}$ have a smaller bias and MSE than that of the $\hat{c}_{M}$ and $\hat{k}_{M}$. In particular, the GA helps to greatly reduce the MSEs of the MLEs, and that means the GA is useful to obtain stable MLEs of the parameters $c$ and $k$ of the BXIID based on PIC samples.

The boxplots of 1000 MLEs of the parameters $c$ and $k$, those were obtained from the simulation, are given in Fig. 1 and 2. Both boxplots indicate that the MLEs, obtained by using the TMLEM, are more spread than that obtained by using the GA. The MLEs of $\hat{c}_{M}$ in Fig. 1 spread over a wide range, and the values could be much larger than the true parameter. The MLEs of $\hat{k}_{M}$ in Fig. 2 are much spread over a wide range compared with that of $\hat{k}_{G}$. All these simulation results show that the TMLEM work unstably to reach the MLEs of the parameters $c$ and $k$, and the GA works better and can be considered as a competitive method instead of the
TMLEM.

\section{Conclusions}

1. In this paper, the TMLEM and GA are used to study the estimation performance of obtaining the maximum likelihood estimates of the parameters of BXIID based on PIC samples. An extensive Monte Carlo simulation was conducted, and the simulation results indicate that the GA based MLEs have a smaller bias and MSE than that MLEs obtained by using the TMLEM.

2. The GA is competitive with the TMLEM to obtain the MLEs of parameters, and therefore we recommend to use the GA to obtain MLEs when the parameters of the BXIID are estimated with using a PIC scheme.

3. Using the GA for other baseline distributions or other testing schemes are also favorable. Other evaluation computation methods, for example the Differential Evaluation, Evolution Strategies and Particle Swarm Optimization methods, could also be helpful to reduce the bias and MSE in parameter estimation. All these topics can be addressed in future studies.

\section{Acknowledgment}

This study is supported by the grant of Ministry of Science and Technology, Taiwan MOST 103-2221-E-032054.

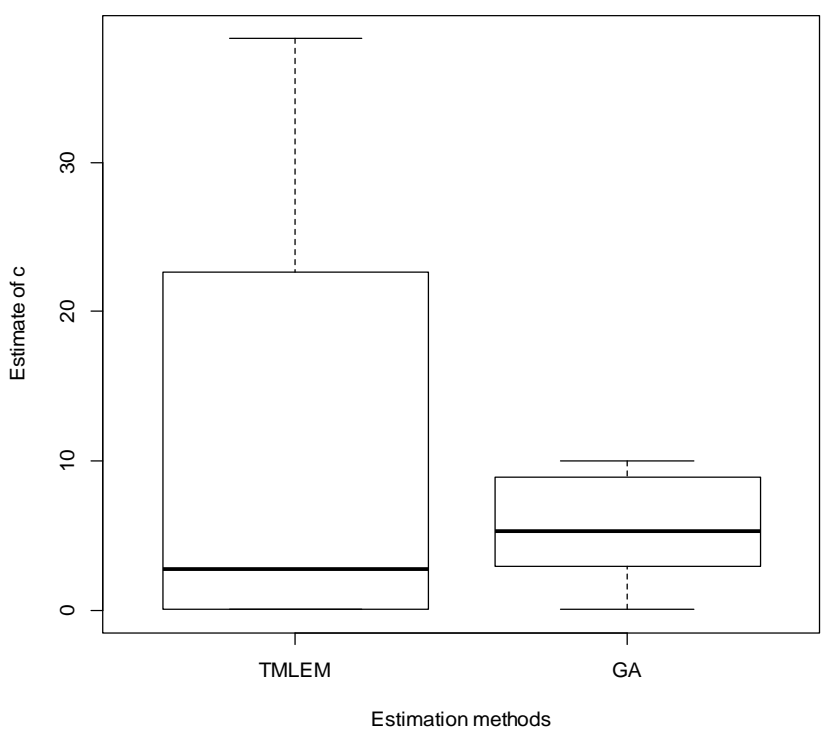

Fig. 1 Boxplot of the MLEs of $c$ obtained by using the TMLEM and GA 


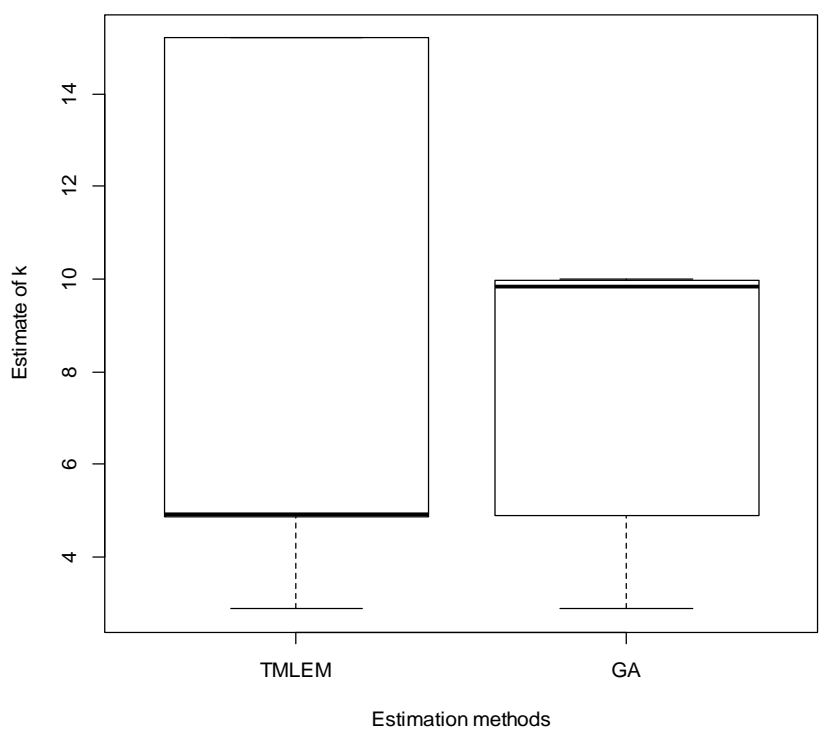

Fig. 2 Box plot of the MLEs of $k$ obtained by using the TMLEM and GA

\section{References}

(1) I.W. Burr, "Cumulative frequency functions”, Annals of Mathematical Statistics, Volume 13, pp. 215-232, 1946.

(2) P.-R. Tadikamalla, "A look at the Burr and related distributions”, International Statistical Review, Volume 48, pp. 373-344, 1980.

(3) D.R. Wingo, "Maximum likelihood methods for fitting the Burr type XII distribution to life test data”, Biometrical Journal, Volume 25, pp. 77-84, 1983.

(4) D.R. Wingo (1993). "Maximum likelihood estimation of Burr XII distribution parameters under type II censoring”, Microelectronics and Reliability, Volume 33, pp. 12511257, 1993.

(5) M.A.M. Ali Mousa, "Empirical Bayes estimators for the Burr type XII accelerated life testing model based on type-2 censored data”, Journal of Statistical Computation and Simulation, Volume 52, no. 2, pp. 95-103, 1995.

(6) P.L. Gupta, R.C. Gupta, S.J. Lvin, “Analysis of failure time data by Burr distribution”, Communications in Statistics-Theory and Methods, Volume 25, pp. 20132024, 1996.

(7) W.J. Zimmer, J.B. Keats and F.K. Wang, "The Burr XII distribution in reliability analysis”, Journal of Quality Technology, Volume 30, pp. 386-394, 1998.
(8) M.A.M. Ali Mousa and Z.F. Jaheen, "Statistical inference for the Burr model based on progressively censored data”, Computers and Mathematics with Applications, Volume 43, pp. 1441-1449, 2002.

(9) S.K. Upadhyay, I.A. Javed and M. Peshwani, "Bayesian analysis of generalized four-parameter Burr distribution via Gibbs sampler”, METRON, Volume LXII, pp. 115135, 2004.

(10) Wu J-W and H.-Y. Yu, "Statistical inference about the shape parameter of the Burr type XII distribution under the failure-censored sampling plan”, Applied Mathematics and Computation, 163: 443-482, 2005.

(11) S.-J. Wu, Y.-J. Chen and C.-T. Chang, "Statistical inference based on progressively censored samples with random removals from the Burr Type XII distribution”, Journal of Statistical Computation and Simulation, Volume 77, pp. 19-27, 2007.

(12) S.-F. Wu, C.-C. Wu, Y.-L. Chen, Y.-R. Yu and Y.P. Lin, "Interval estimation of a two-parameter Burr-XII distribution under progressive censoring”, Statistics, Volume 44, pp. 77-88, 2010.

(13) Y.L. Lio and T.-R. Tsai, "Estimation of $\delta=\mathrm{P}(\mathrm{X}<Y)$ for Burr XII distribution based on the progressively first failure-censored samples”, Journal of Applied Statistics, Volume 39, pp. 309-322, 2012.

(14) M. Aslam, Y.L. Lio, C.-H. Jun, "Repetitive acceptance sampling plans for Burr type XII distribution”, International Journal of Advanced Manufacturing Technology, Volume 68, pp. 495-507, 2013.

(15) A.A. Soliman, A.H. Abd Ellah, N.A. Abou-Elheggag and A.A. Modhesh, "Estimation from Burr type XII distribution using progressive first-failure censored data”, Journal of Statistical Computation and Simulation, Volume 83, no. 12, pp. 2270-2290, 2013.

(16) T.-R. Tsai, Y.L. Lio, N. Jiang, Y.-J. Lin and Y.-Y. Fan, "Economical sampling plans with warranty based on truncated data from Burr type XII distribution”, Journal of the Operational Research Society, to appear.

(17) R. Aggarwala, "Progressively interval censoring: some mathematical results with application to inference", Communications in Statistics-Theory and Methods, Volume 30, pp. 1921-1935, 2001.

(18) C.-K. Ting, "On the mean convergence time of multiparent genetic algorithms without selection", Advances in Artificial Life, Volume 3630, pp. 403-412, 2005.

(19) J. ZHANG, H. Chung and W. L. Lo, “Clustering-based adaptive crossover and mutation probabilities for genetic algorithms”, IEEE Transactions on Evolutionary 
Computation, Volume11, no.3, pp. 326-335, 2007.

(20) Z. Akbari, "A multilevel evolutionary algorithm for optimizing numerical functions", International Journal of Industrial Engineering Computations, Volume 2, Number 2, pp. 419-430, 2011

(21) C. Ferreira, "Gene expression programming: a new adaptive algorithm for solving problems". Complex Systems, Volume 13, Number 2, pp. 87-129, 2001.

(22) M. Nicholson, "Genetic algorithms and grouping problems”, Software: Practice and Experience, Volume 28, Number 10, 1998.

(23) B. Baudry, F. Fleurey, J.M. Jézéquel, and Y. Le Traon, "Automatic test case optimization: a bacteriologic algorithm" IEEE Software, Volume 22, Number 2, pp. 76-82, 2005.

(24) P. Civicioglu, "Transforming geocentric cartesian coordinates to geodetic coordinates by using differential search algorithm". Computers \& Geosciences, Volume 46, pp. 229-247, 2012.

(25) G. Kjellström, "On the efficiency of Gaussian adaptation". Journal of Optimization Theory and Applications, Volume 71, Number 3, pp. 589-597, 1991. 